\title{
p16 Hypermethylation and KRAS Mutation Are Independent Predictors of Cetuximab Plus FOLFIRI Chemotherapy in Patients with Metastatic Colorectal Cancer
}

\author{
Se Hyun Kim, MD ${ }^{1}$ \\ Kyu Hyun Park, PhD² \\ Sang Joon Shin, MD \\ Kang Young Lee, $\mathrm{MD}^{4}$ \\ Tae II Kim, MD ${ }^{3}$ \\ Nam Kyu Kim, MD 4 \\ Sun Young Rha, $\mathrm{MD}^{3}$ \\ Jae Kyung Roh, $\mathrm{MD}^{3}$ \\ Joong Bae Ahn, MD, PhD'2,3
}

${ }^{1}$ Department of Internal Medicine, Seoul National University Bundang Hospital, Seongnam, Institute for Cancer Research, College of Medicine, Yonsei University, Seoul, Departments of ${ }^{3}$ Internal Medicine and ${ }^{4}$ Surgery, Yonsei University

College of Medicine, Seoul, Korea

Correspondence: Joong Bae Ahn, MD, PhD Department of Internal Medicine, Institute for Cancer Research, Yonsei Cancer Center, Yonsei University College of Medicine, 50-1 Yonsei-ro, Seodaemun-gu, Seoul 03722, Korea Tel: 82-2-2228-8134

Fax: 82-2-393-3652

E-mail: vvswm513@yuhs.ac

Received November 2, 2014

Accepted March 11, 2015

Published Online April 24, 2015

*This study was presented in part at the 2012 ESMO meeting, 29 September to 2 October 2012, in Vienna, Austria.

\begin{abstract}
Purpose
Hypermethylation of the $\mathrm{CpG}$ island of $p 16^{1 \mathrm{NK} 4 a}$ occurs in a significant proportion of colorectal cancer (CRC). We aimed to investigate its predictive role in CRC patients treated with 5-fluorouracil, leucovorin, irinotecan (FOLFIRI), and cetuximab.
\end{abstract}

\section{Materials and Methods}

Pyrosequencing was used to identify KRAS mutation and hypermethylation of $6 \mathrm{CpG}$ island loci ( $p 16, p 14$, MINT1, MINT2, MINT31, and $h M L H 1)$ in DNA extracted from formalin-fixed paraffin-embedded specimens. Logistic regression and Cox regression were performed for analysis of the relation between methylation status of $\mathrm{CpG}$ island methylator phenotype (CIMP) markers including $p 16$ and clinical outcome.

\section{Results}

Hypermethylation of the $p 16$ gene was detected in 14 of 49 patients (28.6\%) and showed significant association with KRAS mutation (Fisher exact, $\mathrm{p}=0.01$ ) and CIMP positivity (Fisher exact, $p=0.002)$. Patients with $p 16$-unmethylated tumors had significantly longer time to progression (TTP; median, 9.0 months vs. 3.5 months; log-rank, $p=0.001$ ) and overall survival (median, 44.9 months vs. 16.4 months; log-rank, $p=0.008$ ) than those with $p 16$-methylated tumors. Patients with both KRAS and $p 16$ aberrancy $(n=6)$ had markedly shortened TTP (median, 2.8 months) compared to those with either KRAS or $p 16$ aberrancy ( $\mathrm{n}=11$; median, 8.6 months; $p=0.021$ ) or those with neither ( $n=32$; median, 9.0 months; $\mathrm{p}<0.0001$ ). In multivariate analysis, KRAS mutation and $p 16$ methylation showed independent association with shorter TTP (KRAS mutation: hazard ratio [HR], 3.21; $p=0.017$; p16 methylation: $\mathrm{HR}, 2.97 ; \mathrm{p}=0.027)$.

\section{Conclusion}

Hypermethylation of $p 16$ was predictive of clinical outcome in metastatic CRC patients treated with cetuximab and FOLFIRI, irrespective of KRAS mutation.

\section{Key words}

Colorectal neoplasms, p16, CIMP, Methylation, KRAS

\section{Introduction}

Colorectal cancer (CRC) is a disease of epigenetic as well as genetic aberrancies. These genetic and epigenetic aber- rances are not uniformly present in all patients as in a classic model of colon cancer evolution (adenoma-carcinoma sequence) [1]. Recent studies of colorectal carcinogenesis show that CRC is a heterogeneous disease arising from multiple pathways [2]. Knowledge of key molecular findings of 
each different pathway is not only helpful in explaining clinical outcomes but may also lead to more efficacious and individualized therapies.

The KRAS mutation is a validated biomarker of response to anti-epidermal growth factor receptor (EGFR) antibodies (cetuximab and panitumumab) [3,4]. In prospective randomized trials, the tumor mutation status of codons 12 and 13 of the KRAS gene was predictive for the activity of cetuximab combined with FOLFOX (oxaliplatin/leucovorin/5-fluorouracil) or FOLFIRI (irinotecan/leucovorin/5-fluorouracil) [5]. Therefore, performance of KRAS mutation analysis is mandatory before making treatment decisions. Regarding the prognostic role of KRAS gene, a prior international study found that KRAS mutations generally confer a worse prognosis [6]. However, conflicting results have been reported from analysis of recent large prospective trials [7].

CRCs can also be grouped according to epigenetic alterations, such as DNA methylation status. CpG island methylator phenotype (CIMP) is a distinct group with an increased frequency of aberrant promoter hypermethylation at specific loci. The "classic" CIMP markers (p16, MINT1, MINT2, MINT31, and $h M L H 1)$, which were initially suggested by Toyota et al. [8], defined two groups of CRC. In the study, CIMP positive CRCs had more frequent KRAS but fewer TP53 mutations than CIMP negative CRCs. The close association between CIMP and KRAS mutations as well as BRAF mutations was further reported in subsequent studies with classic markers $[9,10]$. The unique dependency on RAS/RAF pathway in CIMP CRCs might be predictive of anti-EGFR treatment.

The INK4a/ARF/INK4b locus (also known as CDKN2A and $C D K N 2 B$ ) on chromosome 9p21 encodes three genes (ARF, $p 15^{I N K 4 b}$, and $p 16^{I N K 4 a}$ ) [11]. Among them, the $p 16$ gene encodes a G1 cyclin-dependent kinase (CDK) inhibitor that binds to and inactivates CDK4/6. Expression of p16 inhibits CDK4/ 6 mediated phosphorylation of retinoblastoma and results in G1 arrest in tumor cells [12]. The cell cycle arrest mediated by p16 upregulation is thought to be an important barrier to RAS activated oncogenic stress in colonic epithelial cells, termed oncogene-induced senescence [13]. In CRCs, inactivation of $\mathrm{p} 16$ is preferentially mediated by promoter hypermethylation of the $p 16$ gene, one of the classic panels of CIMP [8,12]. In previous studies, alteration of p16, either by promoter hypermethylation or loss of expression, was associated with poor prognosis in patients with CRC [14-16]. In addition, a preclinical study reported that $p 16$ gene hypermethylation was associated with decreased response to irinotecan in colon cancer cell lines and a demethylating agent, 5-azacytidine, enhanced the anti-cancer effect [17].

In this study, we retrospectively evaluated the ability of CIMP status and $p 16$ gene hypermethylation status to predict best objective response (BOR), time to progression (TTP), and overall survival (OS) in CRC patients treated with cetuximab-FOLFIRI (E-FOLFIRI) chemotherapy.

\section{Materials and Methods}

\section{Patient characteristics}

We included 49 patients with metastatic or recurrent CRC who were treated with 5-fluorouracil, leucovorin, irinotecan, and cetuximab (E-FOLFIRI) as first-line (22 patients) or second-line (27 patients) therapy. All patients were treated at Severance Hospital of Yonsei University from January 2005 to January 2011. Clinical data were obtained from electronic medical records of Severance Hospital and survival data were retrieved from the tumor registry at Yonsei Cancer Center. Exclusion criteria included co-existing malignancies (except for non-melanoma skin cancer or in situ cervical cancer), cancer other than adenocarcinoma, and lack of availability of formalin-fixed paraffin-embedded (FFPE) tumor tissue. This study was approved by the institutional review board (IRB) at Yonsei University Severance Hospital (Seoul, Korea).

\section{Treatment and efficacy assessment}

E-FOLFIRI chemotherapy consisted of weekly cetuximab (initial dose $400 \mathrm{mg} / \mathrm{m}^{2}$ intravenously [IV] over 2 hours, and $250 \mathrm{mg} / \mathrm{m}^{2}$ IV weekly, over 1 hour, thereafter) and biweekly FOLFIRI (irinotecan $180 \mathrm{mg} / \mathrm{m}^{2} \mathrm{IV}$ on day 1, leucovorin 200 $\mathrm{mg} / \mathrm{m}^{2} \mathrm{IV}$ on day 1, 5-fluorouracil [5-FU] $400 \mathrm{mg} / \mathrm{m}^{2} \mathrm{IV}$ bolus on day 1 followed by $2,400 \mathrm{mg} / \mathrm{m}^{2} \mathrm{IV}$ over 46 hours, every 2 weeks). FOLFIRI was administered after 1 hour of cetuximab infusion. Treatment was continued until disease progression or unacceptable toxicity. Tumor response was evaluated after four cycles (every 8 weeks) by computed tomography scan and classified according to Response Evaluation Criteria in Solid Tumors (RECIST) criteria ver. 1.1.

\section{DNA methylation and KRAS mutation analysis}

Genomic DNA from FFPE tissue was extracted using QIAamp DNA FFPE tissue kit (Qiagen, Valencia, CA). DNA extracted from FFPE tissue was used to evaluate the methylation status of six CpG islands (MINT1, MINT2, MINT31, $h M L H 1, p 16$, and $p 14)$. Bisulfite treatment of DNA was performed before pyrosequencing using an EZ Methylation kit (Zymo Research, Orange, CA) according to the manufacturer's protocol. Pyrosequencing was performed using the PyroMark Q24 instrument (Qiagen) according to the manufacturer's protocol. The detailed protocol for pyrosequencing 
of DNA methylation has been described in previous reports [10]. The methylation status of $\mathrm{CpG}$ island markers was considered to be methylation positive using a threshold value of $15 \%$ (negative, methylation level $<15 \%$; positive, methylation level $\geq 15 \%$ ). A tumor was considered CIMP positive if two or more CIMP markers were methylation positive.

Mutation status of KRAS gene was also determined with pyrosequencing assays. Mutations of codons 12 and 13 were determined using the PyroMark Q24 instrument (Qiagen) [10].

\section{Statistical analysis}

Differences in BOR rates between the two groups (CIMP positive vs. negative; $p 16$ methylated vs. unmethylated) were analyzed using a two-sided Fisher exact test. The TTP and the OS were estimated using the Kaplan-Meier method and compared using the log-rank test. A Cox proportional hazard model was used to examine the independent contribution of each variable that was considered to be a potential predictive variable for survival in univariate analysis. Regression model included variables that showed significant association with survival in univariate analysis and three variables of interest: age, sex, and CIMP status. A value of $\mathrm{p}<0.05$ was considered significant, and all resulting p-values were two sided. Analyses were performed using SPSS for Windows ver. 18.0 (SPSS Inc., Chicago, IL).

\section{Results}

\section{Patient characteristics}

A total of 49 CRC patients treated with E-FOLFIRI were selected based on sample availability. The baseline characteristics of the patients are summarized in Table 1.p16 gene hypermethylation was observed in 14 of 49 tumors (28.6\%). There was no significant difference in patient age, Eastern Cooperative Oncology Group (ECOG) performance status, histology, location of primary tumor, and prior chemotherapy between $p 16$ methylated and $p 16$ unmethylated tumors. Metastatic disease and recurrent disease at the time of treatment accounted for $77.1 \%$ and $22.9 \%$ of patients without $p 16$ methylation and $35.7 \%$ and $64.3 \%$ in $p 16$ methylation, respectively ( $\mathrm{p}=0.006$ ). Mutations in KRAS codons 12 or 13 were significantly more common in p16-methylated tumors $(42.9 \%)$ than in $p 16$-unmethylated tumors $(8.6 \%, \mathrm{p}=0.01)$. $p 16$ methylation showed significant association with CIMP $(\mathrm{p}=0.002)$.
Table 1. Baseline characteristics

\begin{tabular}{|c|c|c|c|}
\hline & $\begin{array}{l}\text { p16 unmethylated } \\
\qquad(\mathrm{n}=35)\end{array}$ & $\begin{array}{c}p 16 \text { methylated } \\
(\mathrm{n}=14)\end{array}$ & p-value ${ }^{a}$ \\
\hline \multicolumn{4}{|l|}{ Sex } \\
\hline Male & $21(60.0)$ & $9(64.3)$ & \multirow[t]{2}{*}{1.00} \\
\hline Female & $14(40.0)$ & $5(35.7)$ & \\
\hline $\begin{array}{l}\text { Median age } \\
\text { (range, yr) }\end{array}$ & $59(39-69)$ & $59(27-77)$ & 0.94 \\
\hline \multicolumn{4}{|l|}{ ECOG PS } \\
\hline 0 & $23(65.7)$ & $9(64.3)$ & \multirow[t]{2}{*}{0.92} \\
\hline 1 & $12(34.3)$ & $5(35.7)$ & \\
\hline \multicolumn{4}{|l|}{ Histology } \\
\hline $\mathrm{WD} / \mathrm{MD}$ & $31(88.6)$ & $12(85.7)$ & \multirow[t]{2}{*}{0.78} \\
\hline PD/Mucinous & 4 (11.4) & 2 (14.3) & \\
\hline \multicolumn{4}{|l|}{ Tumor location } \\
\hline Proximal & $8(22.9)$ & $4(28.6)$ & \multirow[t]{2}{*}{0.72} \\
\hline Distal & $27(77.1)$ & $10(71.4)$ & \\
\hline \multicolumn{4}{|l|}{ Disease status } \\
\hline Metastatic & $27(77.1)$ & $5(35.7)$ & \multirow[t]{2}{*}{0.006} \\
\hline Recurrent & $5(22.9)$ & $9(64.3)$ & \\
\hline \multicolumn{4}{|l|}{ Metastatic site } \\
\hline Liver & $24(68.6)$ & $7(50)$ & \multirow[t]{4}{*}{0.54} \\
\hline Lung & $2(5.7)$ & $1(7.1)$ & \\
\hline Peritoneum & $3(8.6)$ & $1(7.1)$ & \\
\hline Others & $6(17.1)$ & $5(35.7)$ & \\
\hline \multicolumn{4}{|c|}{ Prior chemotherapy } \\
\hline 0 & 17 (48.6) & $5(35.7)$ & \multirow[t]{2}{*}{0.41} \\
\hline 1 & $18(51.4)$ & $9(64.3)$ & \\
\hline \multicolumn{4}{|l|}{ KRAS } \\
\hline Wild type & $32(91.4)$ & $8(57.1)$ & \multirow[t]{2}{*}{0.01} \\
\hline Mutant & $3(8.6)$ & $6(42.9)$ & \\
\hline \multicolumn{4}{|l|}{ CIMP } \\
\hline CIMP negative & $28(80.0)$ & $4(28.6)$ & \multirow[t]{2}{*}{0.002} \\
\hline CIMP positive & $7(20.0)$ & $10(71.4)$ & \\
\hline
\end{tabular}

Values are presented as number (\%). ECOG PS, Eastern Cooperative Oncology Group performance status; WD, well differentiated; $\mathrm{MD}$, moderately differentiated; $\mathrm{PD}$, poorly differentiated; CIMP, CpG island methylator phe-

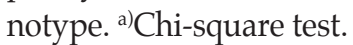

\section{Tumor response and survival according to $p 16$ methyla- tion and CIMP}

Among 49 evaluable patients, 44 patients had one or more measurable lesions and five patients had no measurable disease. The BOR (complete response+partial response) rate by RECIST was $68.8 \%$ in patients with unmethylated $p 16$ (22/32; 95\% confidence interval [CI], 51.5 to 84.8$)$ and $33.3 \%$ (4/12; 95\% CI, 9.1 to 62.5 ) in patients with methylated $p 16$ (Fisher exact test, $\mathrm{p}=0.045$ ) (Table 2). Compared with CIMP negative, patients with CIMP positive tumors had a lower response 
Table 2. Best objective response according to $p 16$ methylation and CIMP status

\begin{tabular}{lcccc} 
& $p 16$ methylated $(\mathbf{n}=\mathbf{1 4})$ & $p 16$ unmethylated $(\mathbf{n}=\mathbf{3 5})$ & CIMP positive $(\mathbf{n}=\mathbf{1 7})$ & \multicolumn{2}{c}{ CIMP negative $(\mathbf{n}=\mathbf{3 2})$} \\
CR & 0 & $2(5.7)$ & 0 & $2(6.3)$ \\
PR & $4(28.6)$ & $20(57.1)$ & $6(35.3)$ & $18(56.3)$ \\
SD & $3(21.4)$ & $7(20)$ & $4(23.5)$ & $6(18.5)$ \\
PD & $5(35.7)$ & $3(8.6)$ & $5(29.4)$ & $3(9.4)$ \\
UM & $2(14.3)$ & $3(8.6)$ & $2(11.8)$ & $3(9.4)$ \\
\hline
\end{tabular}

Values are presented as number $(\%)$. CIMP, CpG island methylator phenotype; $\mathrm{CR}$, complete response; PR, partial response; $\mathrm{SD}$, stable disease; PD, progressive disease; UM, unmeasurable.

A

B
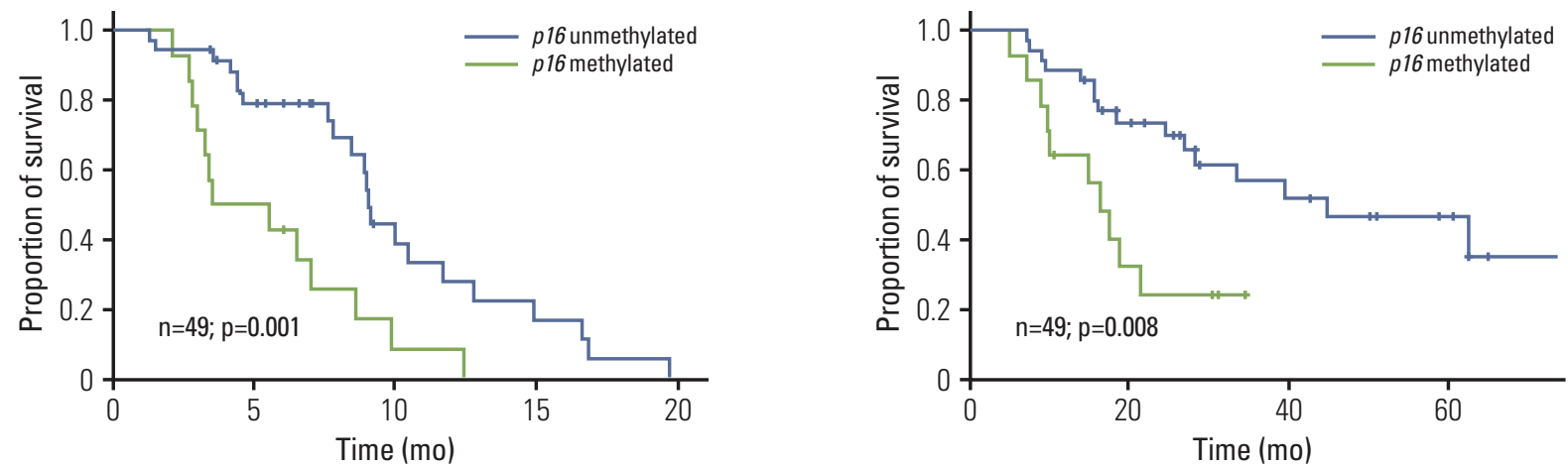

C
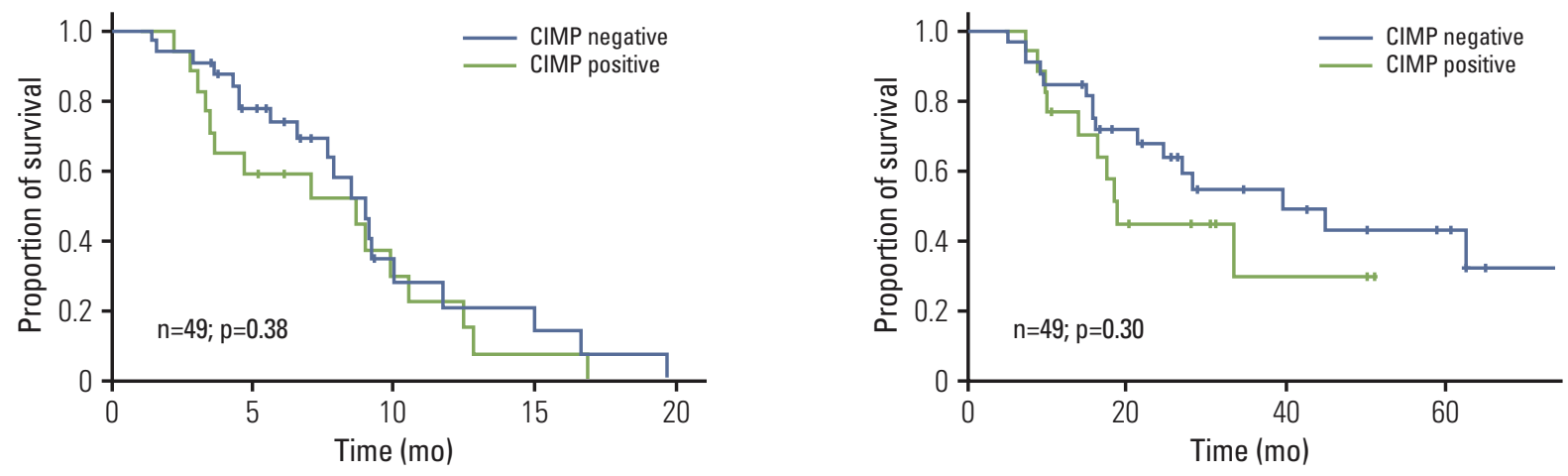

Fig. 1. Kaplan-Meier plots for time to progression (A, C) and overall survival (B, D) according to $p 16$ methylation status and $\mathrm{CpG}$ island methylator phenotype (CIMP) status. CIMP positive denotes tumors with two or more methylation sites in six $\mathrm{CpG}$ islands. All p-values correspond to the log-rank test.

rate $(40 \%$ [6/15; $95 \% \mathrm{CI}, 16.7$ to 69.2$]$ vs. $69 \%$ [20/29; $95 \% \mathrm{CI}$, 50.0 to 85.2$]$ ), but this was not statistically significant (Fisher exact test, $\mathrm{p}=0.064$ ).

Median TTP was 9.0 months (95\% CI, 8.8 to 9.3) in the unmethylated $p 16$ group and 3.5 months (range, 0.0 to 7.4 months) in the methylated $p 16$ group (hazard ratio [HR],
3.15; 95\% CI, 1.52 to 6.54; log-rank, $\mathrm{p}=0.001$ ) (Fig. 1A). In the unmethylated $p 16$ group, 12 of 35 patients $(34.3 \%)$ with unresectable disease were converted to resectable status and underwent surgery, while, on the contrary, none of the methylated $p 16$ patients was able to undergo surgery. With a median follow-up period of 21.4 months (range, 5.1 to 74.3 
Table 3. Multivariate analysis for time to progression in colorectal cancer patients treated with E-FOLFIRI

\begin{tabular}{|c|c|c|c|}
\hline Variable & No. $(n=49)$ & Hazard ratio (95\% CI) & p-value \\
\hline $\begin{array}{l}\text { Age (yr) } \\
<65 \\
\geq 65\end{array}$ & $\begin{array}{l}36 \\
13\end{array}$ & $1.24(0.45-3.44)$ & 0.68 \\
\hline $\begin{array}{l}\text { Sex } \\
\text { Male } \\
\text { Female }\end{array}$ & $\begin{array}{l}30 \\
19\end{array}$ & $1.11(0.44-2.79)$ & 0.83 \\
\hline $\begin{array}{l}\text { Histology } \\
\text { WD/MD } \\
\text { PD/Mucinous }\end{array}$ & $\begin{array}{r}43 \\
6\end{array}$ & $1.89(0.71-5.08)$ & 0.21 \\
\hline $\begin{array}{l}\text { Disease status } \\
\text { Metastatic } \\
\text { Recurrent }\end{array}$ & $\begin{array}{l}32 \\
17\end{array}$ & $1.98(0.79-4.92)$ & 0.14 \\
\hline $\begin{array}{l}\text { Line of treatmer } \\
\text { First line } \\
\text { Second line }\end{array}$ & $\begin{array}{l}22 \\
27\end{array}$ & $1.07(0.46-2.49)$ & 0.88 \\
\hline $\begin{array}{l}\text { p16 methylation } \\
\text { Unmethylated } \\
\text { Methylated }\end{array}$ & $\begin{array}{l}35 \\
14\end{array}$ & $2.97(1.14-7.74)$ & 0.027 \\
\hline $\begin{array}{l}\text { CIMP status } \\
\text { Negative } \\
\text { Positive }\end{array}$ & $\begin{array}{l}32 \\
17\end{array}$ & $0.58(0.23-1.45)$ & 0.24 \\
\hline $\begin{array}{l}\text { KRAS mutation } \\
\text { Wild type } \\
\text { Mutant }\end{array}$ & $\begin{array}{r}40 \\
9\end{array}$ & $3.21(1.23-8.38)$ & 0.017 \\
\hline
\end{tabular}

$\mathrm{WD}$, well differentiated; $\mathrm{MD}$, moderately differentiated; PD, poorly differentiated; CIMP, CpG island methylator phenotype.

months), median OS was 44.9 months (95\% CI, 18.1 to 71.7) in the unmethylated $p 16$ group and 16.4 months (range, 12.0 to 20.7 months) in the methylated $p 16$ group (HR, 2.95; 95\% CI, 1.28 to 6.81; log-rank, $\mathrm{p}=0.008$ ) (Fig. 1B). In our cohort, however, CIMP status was neither a significant predictor of TTP nor of OS ( $p=0.38$ and $p=0.30$, respectively) (Fig. $1 \mathrm{C}$ and D). After correcting for significant predictive factors for TTP by multivariate analysis, $p 16$ methylation was associated with poorer TTP ( $\mathrm{p}=0.027 ; \mathrm{HR}, 2.97 ; 95 \% \mathrm{CI}, 1.14$ to 7.74 ), but not with OS $(\mathrm{p}=0.071)$ (Table 3$)$.

\section{Subgroup analysis stratified by KRAS mutation and $p 16$ methylation}

For a more detailed analysis of response to E-FOLFIRI treatment, we classified patients according to the number of genetic (KRAS mutation) and epigenetic aberrancies ( $p 16$ methylation). Response rate significantly decreased as the

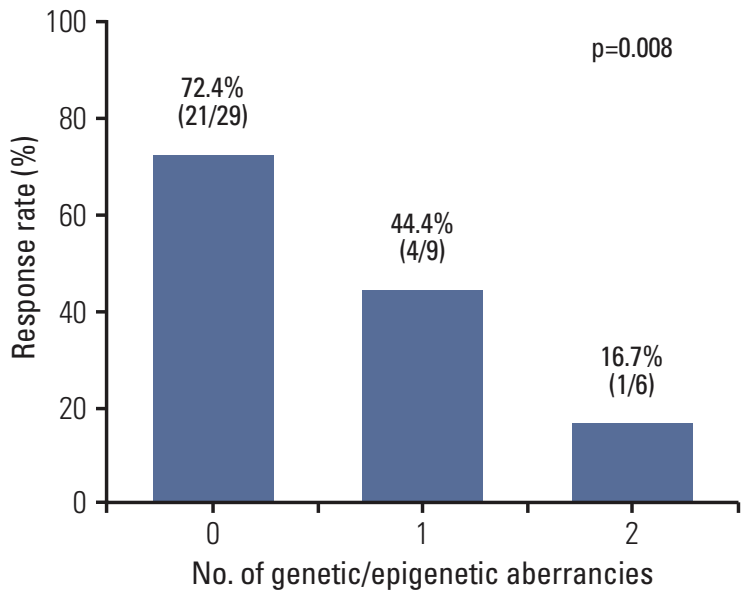

Fig. 2. Response rates according to number of aberrancies (KRAS mutation or $p 16$ methylation). p-value corresponds to the linear-by-linear association test for trend.

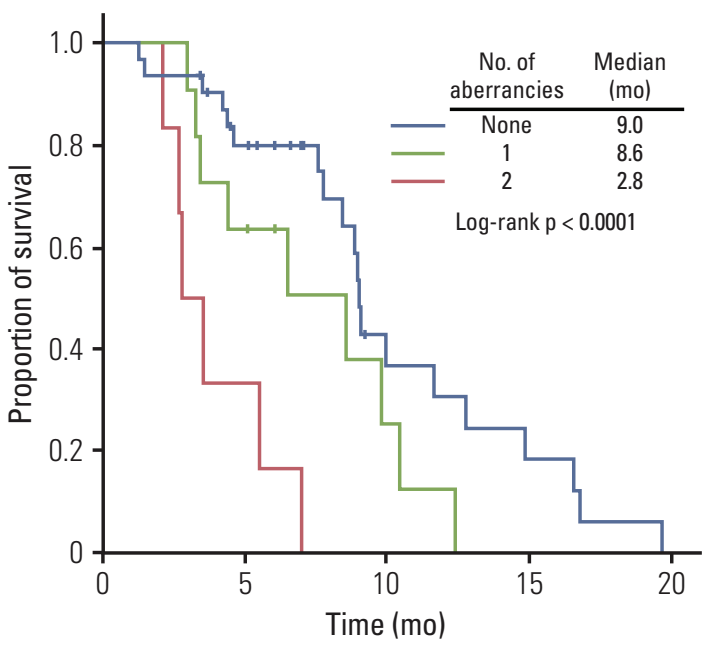

Fig. 3. Kaplan-Meier plot for time to progression according to number of aberrancies (KRAS mutation or $p 16$ methylation). p-value corresponds to the log-rank test.

number of aberrancies increased (Fig. 2). The univariate odds ratio of response between the no aberrancy group and 2 aberrancy group was 13.12 (95\% CI, 1.32 to $130.42 ; \mathrm{p}=0.028$ ). Median TTPs of no aberrancy group, 1 aberrancy group, and 2 aberrancy group were 9.0 months, 8.6 months, and 2.8 months, respectively (log-rank, $\mathrm{p}<0.0001$ ). The KaplanMeier curve for TTP of each group showed a trend toward worse outcome (Fig. 3). 


\section{Discussion}

The primary aim of this study was to explore the use of methylation status in predicting outcome after E-FOLFIRI therapy for metastatic CRC. We have shown that epigenetic alteration of $p 16$ is independently associated with poorer outcome in these patients. In addition, patients with both KRAS mutation and $p 16$ methylation had remarkably poor prognosis despite E-FOLFIRI treatment. These findings suggest that epigenetic alteration, in addition to genetic aberrancies such as KRAS mutation, may be helpful in defining which patients are likely to benefit from systemic treatment in CRC patients.

It should be noted that CRC patients with both $p 16$ methylation and KRAS mutation have shorter survival than those with either one alone or no aberrancies. The role of p16 as a tumor suppressor is well established in a variety of human cancers [11,12]. It has been postulated that p16 plays a significant role in the signal transduction of the RAS/RAF pathway and acts as a barrier to oncogenic stimulation. In CRCs, Esteller et al. [14] reported shorter survival of CRC patients with KRAS mutation and/or p16 methylation after surgical resection compared to those with neither of those alterations. In a variety of tumors with RAS / RAF activation, such as melanoma, pancreatic cancer, and malignant astrocytoma, frequent concomitant loss of p16 has been observed and conferred resistance to chemotherapeutic agents [18]. Recently, in a preclinical study that utilized CRC cell lines, p16 demethylation by 5-azacytidine enhanced cytotoxicity of irinotecan treatment [17]. In addition, inhibition of CDK4/ 6 with PD0332991, a novel selective CDK 4/ 6 inhibitor, instead of p16 could be another potential targeted agent. It has already demonstrated antitumor activity in a human CRC xenograft model and showed significant clinical activity in hormone receptor positive breast cancer patients [19]. Therefore, combining demethylating agents or PD0332991 with chemotherapeutic agents or RAS/RAF pathway inhibitor might be a rational approach to overcome resistance and improve outcomes in patients with this aggressive tumor type.

CIMP subtype was not a powerful predictor of clinical outcome in our study. CIMP subtype did not show significant association with TTP and OS, despite a trend toward lower response rate in CIMP positive. Previous studies regarding molecular characteristics of CIMP subtype have shown that CIMP positive tumors are characterized by a high frequency of KRAS and BRAF mutations $[9,10,20]$. On the basis of this molecular characteristic of CIMP, it has been suggested that CIMP status might be a possible predictive marker for antiEGFR therapy. In addition, patients with CIMP positive tumors were associated with mismatch repair deficiency and tend to show a worse response to 5-FU chemotherapy [21].
A study by Ogino et al. [22] reported that CIMP status predicted treatment response and survival in microsatellite stable CRC patients after 5-FU and irinotecan based combination chemotherapy. For the subtype specific personalized therapeutic approach in CRC, further studies are needed to elucidate the exact mechanism and overcome the inherent chemoresistance of CIMP subtype.

Biomarker identification in cancer tissue is a crucial step in development of personalized treatment. The clinical impact and usefulness of biomarkers are dependent on their stability and reproducibility. In contrast to the relatively low stability of protein or RNA based biomarkers, the advantage of DNA methylation marker is stability over time. DNA methylation in gene promoters remains stable over years in cancer cell lines and xenograft models [23]. A recent study demonstrated that gene expression of unmethylated promoters is quite variable but gene expression is stably repressed when the promoters are methylated [24]. Moreover, the reversibility of DNA methylation by hypomethylating agents suggests that they are not only surrogate markers of response but also potential targets for treatment. This point may be addressed by an ongoing clinical trial (Clinical Trial.gov identifier No. NCT01105377).

We note that our study had several limitations. First, study results must be interpreted with caution because of the small number of patients who were analyzed without a control arm. Therefore, these results should be validated in a larger setting in prospective cohorts. Second, BRAF mutation analysis was not performed in our study. As KRAS and BRAF mutations are mutually exclusive [20], poor survival in patients with methylated $p 16$ and KRAS mutation is not affected by $B R A F$ status. In addition, recent studies suggest that the less common mutation of KRAS (in exon 3 or 4) and NRAS predicted a lack of response in patients who received anti-EGFR antibody [25]. Last, we utilized "classic" CIMP panels because various groups using this panel have found consistent results in CRC patients $[8,10,20]$. However, the absence of a consensus panel defining the CIMP subgroup is a hurdle to be overcome with future investigations.

\section{Conclusion}

In conclusion, we found that hypermethylation of $p 16$ was predictive of clinical outcome in CRC patients treated with E-FOLFIRI, irrespective of KRAS mutation status. Although CIMP status was not predictive of E-FOLFIRI response, there was a trend toward poor responsiveness. These findings should be validated in further prospective trials for individualized cancer treatment. 


\section{Conflicts of Interest}

Conflict of interest relevant to this article was not reported.

\section{Acknowledgments}

This study was supported by a faculty research grant of Yonsei University College of Medicine for 2010 (6-2010-0188).

\section{References}

1. Fearon ER, Vogelstein B. A genetic model for colorectal tumorigenesis. Cell. 1990;61:759-67.

2. Jass JR. Classification of colorectal cancer based on correlation of clinical, morphological and molecular features. Histopathology. 2007;50:113-30.

3. Kelley RK, Wang G, Venook AP. Biomarker use in colorectal cancer therapy. J Natl Compr Canc Netw. 2011;9:1293-302.

4. Schmoll HJ, Van Cutsem E, Stein A, Valentini V, Glimelius B, Haustermans K, et al. ESMO Consensus Guidelines for management of patients with colon and rectal cancer. a personalized approach to clinical decision making. Ann Oncol. 2012;23: 2479-516.

5. Van Cutsem E, Kohne CH, Lang I, Folprecht G, Nowacki MP, Cascinu S, et al. Cetuximab plus irinotecan, fluorouracil, and leucovorin as first-line treatment for metastatic colorectal cancer: updated analysis of overall survival according to tumor KRAS and BRAF mutation status. J Clin Oncol. 2011;29: 2011-9.

6. Andreyev HJ, Norman AR, Cunningham D, Oates JR, Clarke PA. Kirsten ras mutations in patients with colorectal cancer: the multicenter "RASCAL" study. J Natl Cancer Inst. 1998;90: 675-84.

7. Hutchins G, Southward K, Handley K, Magill L, Beaumont C, Stahlschmidt J, et al. Value of mismatch repair, KRAS, and BRAF mutations in predicting recurrence and benefits from chemotherapy in colorectal cancer. J Clin Oncol. 2011;29: 1261-70.

8. Toyota M, Ohe-Toyota M, Ahuja N, Issa JP. Distinct genetic profiles in colorectal tumors with or without the $\mathrm{CpG}$ island methylator phenotype. Proc Natl Acad Sci U S A. 2000;97: 710-5.

9. Barault L, Charon-Barra C, Jooste V, de la Vega MF, Martin L, Roignot $\mathrm{P}$, et al. Hypermethylator phenotype in sporadic colon cancer: study on a population-based series of 582 cases. Cancer Res. 2008;68:8541-6.

10. Ahn JB, Chung WB, Maeda O, Shin SJ, Kim HS, Chung HC, et al. DNA methylation predicts recurrence from resected stage III proximal colon cancer. Cancer. 2011;117:1847-54.

11. Kim WY, Sharpless NE. The regulation of INK4/ ARF in cancer and aging. Cell. 2006;127:265-75.

12. Liggett WH Jr, Sidransky D. Role of the p16 tumor suppressor gene in cancer. J Clin Oncol. 1998;16:1197-206.

13. Bennecke M, Kriegl L, Bajbouj M, Retzlaff K, Robine S, Jung A, et al. Ink4a/ Arf and oncogene-induced senescence prevent tumor progression during alternative colorectal tumorigene- sis. Cancer Cell. 2010;18:135-46.

14. Esteller M, Gonzalez S, Risques RA, Marcuello E, Mangues R, Germa JR, et al. K-ras and p16 aberrations confer poor prognosis in human colorectal cancer. J Clin Oncol. 2001;19: 299-304.

15. Karamitopoulou E, Zlobec I, Koumarianou A, Patsouris ES, Peros G, Lugli A. Expression of p16 in lymph node metastases of adjuvantly treated stage III colorectal cancer patients identifies poor prognostic subgroups: a retrospective analysis of biomarkers in matched primary tumor and lymph node metastases. Cancer. 2010;116:4474-86.

16. Bihl MP, Foerster A, Lugli A, Zlobec I. Characterization of CDKN2A(p16) methylation and impact in colorectal cancer: systematic analysis using pyrosequencing. J Transl Med. 2012; 10:173.

17. Crea F, Giovannetti E, Cortesi F, Mey V, Nannizzi S, Gallegos Ruiz MI, et al. Epigenetic mechanisms of irinotecan sensitivity in colorectal cancer cell lines. Mol Cancer Ther. 2009;8: 1964-73.

18. Gallagher SJ, Thompson JF, Indsto J, Scurr LL, Lett M, Gao BF, et al. p16INK4a expression and absence of activated B-RAF are independent predictors of chemosensitivity in melanoma tumors. Neoplasia. 2008;10:1231-9.

19. Finn RS, Crown JP, Boer K, Lang I, Parikh RJ, Breazna A, et al. Results of a randomized phase 2 study of PD 0332991, a cyclin-dependent kinase (CDK) 4 / 6 inhibitor, in combination with letrozole vs letrozole alone for first line treatment of ER+ / HER2- advanced breast cancer (BC). Ann Oncol. 2012; 23(Suppl 2):ii43-5.

20. Samowitz WS, Albertsen H, Herrick J, Levin TR, Sweeney C, Murtaugh MA, et al. Evaluation of a large, population-based sample supports a $\mathrm{CpG}$ island methylator phenotype in colon cancer. Gastroenterology. 2005;129:837-45.

21. Shen L, Catalano PJ, Benson AB 3rd, O'Dwyer P, Hamilton SR, Issa JP. Association between DNA methylation and shortened survival in patients with advanced colorectal cancer treated with 5-fluorouracil based chemotherapy. Clin Cancer Res. 2007;13:6093-8.

22. Ogino S, Meyerhardt JA, Kawasaki T, Clark JW, Ryan DP, Kulke $\mathrm{MH}$, et al. CpG island methylation, response to combination chemotherapy, and patient survival in advanced microsatellite stable colorectal carcinoma. Virchows Arch. 2007;450:529-37.

23. Markl ID, Cheng J, Liang G, Shibata D, Laird PW, Jones PA. Global and gene-specific epigenetic patterns in human bladder 
cancer genomes are relatively stable in vivo and in vitro over time. Cancer Res. 2001;61:5875-84.

24. Claus R, Lucas DM, Stilgenbauer S, Ruppert AS, Yu L, Zucknick $\mathrm{M}$, et al. Quantitative DNA methylation analysis identifies a single $\mathrm{CpG}$ dinucleotide important for ZAP-70 expression and predictive of prognosis in chronic lymphocytic leukemia. J Clin Oncol. 2012;30:2483-91.

25. Douillard JY, Oliner KS, Siena S, Tabernero J, Burkes R, Barugel M, et al. Panitumumab-FOLFOX4 treatment and RAS mutations in colorectal cancer. N Engl J Med. 2013;369: 1023-34. 